COMPARISONS OF LC PROOFSLIP AND MARC TAPE ARRIVAL DATES AT THE UNIVERSITY OF CHICAGO LIBRARY

Charles T. PAYNE: Systems Development Librarian, and Robert S. McGEE: Assistant Systems Development Librarian; University of Chicago Library, Chicago, Illinois

A comparison of arrival dates of 5020 LC proofslips and corresponding MARC magnetic tape records reveals that four-fifths of the MARC records were received the same week as, or earlier than, the proofslips.

The purpose of this study is to determine the timeliness of MARC II records' arrival dates in comparison to the arrival dates of matching LC proofslips. The Acquisitions Department of the University of Chicago Library receives a complete set of cut and punched LC proofsheets (or "LC proofslips") that is used primarily for selection and ordering. In examining potential uses of MARC records in acquisitions processing, the Library Systems Development Office felt that a critical determinant would be the timeliness of MARC records in comparison to the arrival dates of the matching LC proofslips. Accordingly, the study described below was designed to gather data upon which appropriate system design questions might be considered.

It was decided that "arrival date" would be defined as the week in which an arrival occurred, since the initial processing and distribution of incoming LC proofslips is framed within weekly, rather than daily, periods. "Week" was defined as the Monday through Friday workweek. "Arrivals" were defined as deliveries of MARC tapes and LC proofslips by the Library mail service. No attempt was made to influence the normal delivery procedures, or to specialize or hasten identification of these 
materials for priority handling. Arrival weeks were numbered consecutively, the week of March 31 -April 4, 1969, being designated Week 1. MARC tape numbers correspond to arrival week numbers; i.e., MARC tape \#4 arrived during Week 4. Table 1 presents these correspondences.

Table 1. Week Numbers for 15 Weeks of Study

\section{Week Number}

1
2
3
4
5
6
7
8
9
10
11
12
13
14
15

Arrival Week Dates

March 31 - April 4

April 7 -April 11

April 14-April 18

April 21 - April 25

April 28 - May 2

May 5 - May 9

May 12 - May 16

May 19 - May 23

May 26 - May 30

June 2 - June 6

June 9 - June 13

June 16 - June 20

June 23 - June 27

June 30 - July 3

July 7 - July 11

\section{DATA COLLECTION}

Proofslip collection began in Week 2, but in that week only a partial collection was made. In subsequent weeks, complete collections of proofslips bearing the MARC acronym (MARC proofslips) were attempted, so that proofslip data beginning with Week 3 (April 14-18) are more complete. Proofslip collection was terminated in Week 15. Discrepancies between the counts of MARC records and the numbers of MARC proofslips collected have not been accounted for, but possible reasons are discussed in the following section.

Data collection was based upon comparisons of: 1) the weekly printed indexes, in LC card number order, that came with MARC II tapes; and 2) weekly lists of MARC proofslip arrivals. In each incoming batch of LC proofslips, those with MARC notes were separated and their arrival date noted. The MARC proofslips for each week were put in primary order by the first two digits (series number) of the card number, and were secondarily ordered within each series by the serial number following the hyphen, thereby matching the order of LC card numbers in the MARC indexes. These numbers were transcribed to create a weekly list of proofslip arrivals.

Two new lists of LC card numbers were derived each week: 1) a MARC index; and 2) a proofslip list. Weekly each new list was compared with all lists of the other type to identify card number matches. 
Thus, each of the two types of lists was cross-tabulated with all the lists of the other type, showing on all lists which card numbers had been matched, and the week numbers of these matches.

Counts were made of the matches tabulated on each list, and were entered into Table 2. Matches made during a given week are subcounted by series groups $65-68,69$, and the 7 series.

The cumulative percentages of MARC record and proofslips matches were entered into Tables 3 and 4 . Table 3 contains the percentages of matches for any week's proofslips with successive MARC tapes. For example, of the 340 proofslips received in Week 4, 71.2\% matched MARC records received the same week, or earlier, i.e., tapes 4, 3, and 2. Table 4 contains the percentage of matches for any MARC index on successive proofslip lists. For example, of the 768 records on MARC tape number 5 (received in Week 5) 23\% were matched by proofslips received the same week, or earlier, i.e., Weeks 5, 4, 3, and 2 .

\section{ANALYSIS OF RESULTS}

Some patterns of MARC and proofslip arrivals are indicated by the tables. The results in Table 2 show that there is not a one-for-one weekly relationship between proofslip and MARC record arrivals. For example, the 340 MARC proofslips received in Week 4 matched tape records received from Week 2 through Week 10, although the highest number of matches was also in the tape received in Week 4. In later proofslip weeks, however, the highest number of proofslip matches was with tape records received at least one week earlier.

A summary of Table 2 would show that of the 5020 MARC proofslips received during Weeks $3-10,4004$, or $79.8 \%$ were matched to MARC records received the same week or earlier.

In Table 3 , the cumulative percentages of proofslip matches with successive MARC indexes indicate, for several of the weeks, more than a $90 \%$ match with tape records two weeks after proofslip arrivals. Table 3 shows that the percentage of matches for a set of proofslips received in one week with the MARC indexes received the same week or earlier ranges from $48.9 \%$ to $91.6 \%$.

Table 4 shows that the percentage of matches for a MARC tape received in a given week with the proofslips received the same week or earlier ranges from $7.1 \%$ to $49.8 \%$.

For the period of weeks corresponding to tape numbers 3-10, 6335 tape records (from Table 4) and 5020 proofslips (from Table 2 or 3 ) were received. The reason for the discrepancy between the number of MARC records and the number of MARC proofslips is not clear, but is possibly due to the combined effects of basic factors such as the limited period of the study, the difficulties of collecting proofslips in a working environment, and the nature of the manual effort required to list LC card numbers and compare proofslip lists and MARC indexes. 
Table 2. Number of Proofslip Matches with MARC Indexes by Arrival Week and by LC Card Number Subseries

\begin{tabular}{|c|c|c|c|c|c|c|c|c|c|c|c|c|c|c|c|c|}
\hline $\begin{array}{l}\text { Proofslip } \\
\text { Week }\end{array}$ & $\begin{array}{c}\text { LC } \\
\text { Series }\end{array}$ & $\begin{array}{c}\text { Tape } \\
1\end{array}$ & $\begin{array}{c}\text { Tape } \\
2\end{array}$ & $\begin{array}{c}\text { Tape } \\
3\end{array}$ & $\begin{array}{c}\text { Tape } \\
4\end{array}$ & $\begin{array}{c}\text { Tape } \\
5\end{array}$ & $\begin{array}{c}\text { Tape } \\
6\end{array}$ & $\begin{array}{c}\text { Tape } \\
7\end{array}$ & $\begin{array}{c}\text { Tape } \\
8\end{array}$ & $\begin{array}{c}\text { Tape } \\
9\end{array}$ & $\begin{array}{c}\text { Tape } \\
10\end{array}$ & $\begin{array}{c}\text { Tape } \\
11\end{array}$ & $\begin{array}{c}\text { Tape } \\
12\end{array}$ & $\begin{array}{c}\text { Tape } \\
13\end{array}$ & $\begin{array}{c}\text { Tape } \\
14\end{array}$ & $\begin{array}{c}\text { Tape } \\
15\end{array}$ \\
\hline $\begin{array}{l}\text { PS } 2 \\
\# 88\end{array}$ & $\begin{array}{l}65-68 \\
69 \\
7 \text { series }\end{array}$ & $\begin{array}{l}5 \\
2 \\
1\end{array}$ & $\begin{array}{r}25 \\
8 \\
2 \\
\end{array}$ & $\begin{array}{r}13 \\
5 \\
7\end{array}$ & $\begin{array}{l}4 \\
3 \\
1\end{array}$ & $\begin{array}{l}4 \\
3 \\
2\end{array}$ & $\begin{array}{l}0 \\
0 \\
1\end{array}$ & $\begin{array}{l}0 \\
1 \\
0\end{array}$ & $\begin{array}{l}0 \\
0 \\
0\end{array}$ & $\begin{array}{l}0 \\
0 \\
0\end{array}$ & $\begin{array}{l}0 \\
0 \\
0\end{array}$ & $\begin{array}{l}0 \\
0 \\
0\end{array}$ & $\begin{array}{l}0 \\
0 \\
0\end{array}$ & $\begin{array}{l}0 \\
0 \\
0\end{array}$ & $\begin{array}{l}0 \\
0 \\
0\end{array}$ & $\begin{array}{l}0 \\
0 \\
0\end{array}$ \\
\hline Total & & 8 & 35 & 25 & 8 & 9 & 1 & 1 & 0 & 0 & 0 & 0 & 0 & 0 & 0 & 0 \\
\hline $\begin{array}{l}\text { PS } 3 \\
\# 497\end{array}$ & $\begin{array}{l}65-68 \\
69 \\
7 \text { series }\end{array}$ & $\begin{array}{l}6 \\
7 \\
0\end{array}$ & $\begin{array}{l}42 \\
25 \\
16\end{array}$ & $\begin{array}{l}77 \\
65 \\
36\end{array}$ & $\begin{array}{l}37 \\
32 \\
41\end{array}$ & $\begin{array}{r}17 \\
30 \\
8\end{array}$ & $\begin{array}{l}9 \\
9 \\
2\end{array}$ & $\begin{array}{l}2 \\
5 \\
0\end{array}$ & $\begin{array}{l}1 \\
0 \\
0\end{array}$ & $\begin{array}{l}0 \\
1 \\
2\end{array}$ & $\begin{array}{l}0 \\
0 \\
0\end{array}$ & $\begin{array}{l}0 \\
0 \\
0\end{array}$ & $\begin{array}{l}0 \\
0 \\
0\end{array}$ & $\begin{array}{l}0 \\
0 \\
0\end{array}$ & $\begin{array}{l}0 \\
0 \\
0\end{array}$ & $\begin{array}{l}0 \\
0 \\
0\end{array}$ \\
\hline Total & & 13 & 83 & 178 & 110 & 55 & 20 & 7 & $I$ & 3 & 0 & 0 & 0 & 0 & 0 & 0 \\
\hline $\begin{array}{r}\text { PS } 4 \\
\quad \# 340 \\
\end{array}$ & $\begin{array}{l}65-68 \\
69 \\
7 \text { series } \\
\end{array}$ & $\begin{array}{l}0 \\
0 \\
0 \\
\end{array}$ & $\begin{array}{r}14 \\
0 \\
0 \\
\end{array}$ & $\begin{array}{l}35 \\
26 \\
18 \\
\end{array}$ & $\begin{array}{l}57 \\
56 \\
36 \\
\end{array}$ & $\begin{array}{l}19 \\
12 \\
19 \\
\end{array}$ & $\begin{array}{r}12 \\
3 \\
9 \\
\end{array}$ & $\begin{array}{l}1 \\
0 \\
1 \\
\end{array}$ & $\begin{array}{l}3 \\
1 \\
0 \\
\end{array}$ & $\begin{array}{l}2 \\
1 \\
1 \\
\end{array}$ & $\begin{array}{l}1 \\
3 \\
2 \\
\end{array}$ & $\begin{array}{l}0 \\
0 \\
0 \\
\end{array}$ & $\begin{array}{l}0 \\
0 \\
0 \\
\end{array}$ & $\begin{array}{l}0 \\
0 \\
0 \\
\end{array}$ & $\begin{array}{l}0 \\
0 \\
0 \\
\end{array}$ & $\begin{array}{l}0 \\
0 \\
0 \\
\end{array}$ \\
\hline Total & & 0 & 14 & 79 & 149 & 50 & 24 & 2 & 4 & 4 & 6 & 0 & 0 & 0 & 0 & 0 \\
\hline $\begin{aligned} \text { PS } 5 \\
\\
\# 398\end{aligned}$ & $\begin{array}{l}65-68 \\
69 \\
7 \text { series }\end{array}$ & $\begin{array}{l}0 \\
0 \\
0\end{array}$ & $\begin{array}{l}0 \\
0 \\
0\end{array}$ & $\begin{array}{r}14 \\
7 \\
9\end{array}$ & $\begin{array}{l}56 \\
62 \\
49\end{array}$ & $\begin{array}{r}33 \\
21 \\
9\end{array}$ & $\begin{array}{r}35 \\
8 \\
9\end{array}$ & $\begin{array}{l}0 \\
0 \\
1\end{array}$ & $\begin{array}{l}4 \\
4 \\
5 \\
\end{array}$ & $\begin{array}{l}7 \\
3 \\
2 \\
\end{array}$ & $\begin{array}{l}4 \\
2 \\
6\end{array}$ & $\begin{array}{l}0 \\
0 \\
1\end{array}$ & $\begin{array}{l}0 \\
0 \\
0\end{array}$ & $\begin{array}{l}0 \\
0 \\
0\end{array}$ & $\begin{array}{l}0 \\
0 \\
0\end{array}$ & $\begin{array}{l}0 \\
0 \\
0\end{array}$ \\
\hline Total & & 0 & 0 & 30 & 167 & 63 & 52 & 1 & 13 & 12 & 12 & 1 & 0 & 0 & 0 & 0 \\
\hline $\begin{aligned} \text { PS } & 6 \\
& \# 653\end{aligned}$ & $\begin{array}{l}65-68 \\
69 \\
7 \text { series }\end{array}$ & $\begin{array}{l}0 \\
0 \\
0\end{array}$ & $\begin{array}{l}0 \\
0 \\
0\end{array}$ & $\begin{array}{l}0 \\
1 \\
2 \\
\end{array}$ & $\begin{array}{l}29 \\
55 \\
28 \\
\end{array}$ & $\begin{array}{r}108 \\
95 \\
72 \\
\end{array}$ & $\begin{array}{l}77 \\
50 \\
52 \\
\end{array}$ & $\begin{array}{l}3 \\
4 \\
6 \\
\end{array}$ & $\begin{array}{l}5 \\
2 \\
0 \\
\end{array}$ & $\begin{array}{l}2 \\
2 \\
5 \\
\end{array}$ & $\begin{array}{l}3 \\
4 \\
5 \\
\end{array}$ & $\begin{array}{l}1 \\
0 \\
2 \\
\end{array}$ & $\begin{array}{l}0 \\
0 \\
1\end{array}$ & $\begin{array}{l}0 \\
0 \\
0 \\
\end{array}$ & $\begin{array}{l}0 \\
0 \\
0\end{array}$ & $\begin{array}{l}0 \\
0 \\
0\end{array}$ \\
\hline Total & & 0 & 0 & 3 & 112 & 275 & 179 & 13 & 7 & 9 & 12 & 3 & 1 & 0 & 0 & 0 \\
\hline $\begin{array}{r}\text { PS } 7 \\
\# 711\end{array}$ & $\begin{array}{l}65-68 \\
69 \\
7 \text { seried }\end{array}$ & $\begin{array}{l}0 \\
0 \\
0\end{array}$ & $\begin{array}{l}0 \\
0 \\
0\end{array}$ & $\begin{array}{l}0 \\
0 \\
0\end{array}$ & $\begin{array}{l}9 \\
2 \\
5\end{array}$ & $\begin{array}{l}68 \\
29 \\
33 \\
\end{array}$ & $\begin{array}{r}128 \\
133 \\
92 \\
\end{array}$ & $\begin{array}{l}29 \\
54 \\
33 \\
\end{array}$ & $\begin{array}{r}6 \\
9 \\
10 \\
\end{array}$ & $\begin{array}{l}4 \\
1 \\
3\end{array}$ & $\begin{array}{l}2 \\
8 \\
6 \\
\end{array}$ & $\begin{array}{l}0 \\
3 \\
1\end{array}$ & $\begin{array}{l}0 \\
0 \\
2 \\
\end{array}$ & $\begin{array}{l}0 \\
0 \\
0 \\
\end{array}$ & $\begin{array}{l}0 \\
1 \\
0 \\
\end{array}$ & $\begin{array}{l}0 \\
0 \\
0 \\
\end{array}$ \\
\hline Total & & 0 & 0 & 0 & 16 & 130 & 353 & 116 & 25 & 8 & 16 & 4 & 2 & 0 & 1 & 0 \\
\hline $\begin{array}{r}\text { PS } 8 \\
\# 503\end{array}$ & $\begin{array}{l}65-68 \\
69 \\
7 \text { seried }\end{array}$ & $\begin{array}{l}1 \\
0 \\
0\end{array}$ & $\begin{array}{l}0 \\
0 \\
0\end{array}$ & $\begin{array}{l}0 \\
0 \\
0\end{array}$ & $\begin{array}{l}0 \\
0 \\
0\end{array}$ & $\begin{array}{l}5 \\
2 \\
2\end{array}$ & $\begin{array}{l}87 \\
54 \\
37\end{array}$ & $\begin{array}{l}46 \\
49 \\
46\end{array}$ & $\begin{array}{l}29 \\
20 \\
17\end{array}$ & $\begin{array}{r}6 \\
11 \\
1\end{array}$ & $\begin{array}{l}4 \\
4 \\
2\end{array}$ & $\begin{array}{l}0 \\
0 \\
0\end{array}$ & $\begin{array}{l}2 \\
0 \\
0\end{array}$ & $\begin{array}{l}0 \\
0\end{array}$ & $\begin{array}{l}0 \\
0 \\
0\end{array}$ & $\begin{array}{l}0 \\
0 \\
0\end{array}$ \\
\hline Total & & 1 & 0 & 0 & 0 & 9 & 178 & 141 & 66 & 18 & 10 & 0 & 2 & $\frac{1}{1}$ & مـ & ــ \\
\hline $\begin{array}{r}\text { PS } 9 \\
\# 933\end{array}$ & $\begin{array}{l}65-68 \\
69 \\
7 \text { series }\end{array}$ & $\begin{array}{l}0 \\
0 \\
0\end{array}$ & $\begin{array}{l}0 \\
0 \\
0\end{array}$ & $\begin{array}{l}0 \\
0 \\
0\end{array}$ & $\begin{array}{l}0 \\
0 \\
0 \\
\end{array}$ & $\begin{array}{l}0 \\
1 \\
0\end{array}$ & $\begin{array}{r}10 \\
3 \\
1 \\
\end{array}$ & $\begin{array}{l}86 \\
75 \\
49\end{array}$ & $\begin{array}{l}122 \\
107 \\
115\end{array}$ & $\begin{array}{l}52 \\
53 \\
73 \\
\end{array}$ & $\begin{array}{l}34 \\
39 \\
42 \\
\end{array}$ & $\begin{array}{r}5 \\
0 \\
10\end{array}$ & $\begin{array}{l}1 \\
0 \\
4\end{array}$ & $\begin{array}{l}1 \\
0 \\
0\end{array}$ & $\begin{array}{l}0 \\
1 \\
1\end{array}$ & $\begin{array}{l}0 \\
0 \\
0\end{array}$ \\
\hline Total & & 0 & 0 & 0 & 0 & 1 & 14 & 210 & 344 & 178 & 115 & 15 & 5 & 1 & 2 & 0 \\
\hline $\begin{array}{l}\text { PS } 10 \\
\# 985 .\end{array}$ & \begin{tabular}{l|}
$65-68$ \\
69 \\
7 series
\end{tabular} & $\begin{array}{l}0 \\
0 \\
0\end{array}$ & $\begin{array}{l}0 \\
0 \\
0\end{array}$ & $\begin{array}{l}0 \\
0 \\
0\end{array}$ & $\begin{array}{l}0 \\
0 \\
0\end{array}$ & $\begin{array}{l}0 \\
0 \\
0\end{array}$ & $\begin{array}{l}0 \\
0 \\
0\end{array}$ & $\begin{array}{l}5 \\
1 \\
5\end{array}$ & $\begin{array}{l}36 \\
40 \\
23\end{array}$ & $\begin{array}{l}159 \\
180 \\
165\end{array}$ & $\begin{array}{r}91 \\
96 \\
101\end{array}$ & $\begin{array}{l}8 \\
5 \\
7\end{array}$ & $\begin{array}{l}1 \\
1 \\
1\end{array}$ & $\begin{array}{l}0 \\
1 \\
0\end{array}$ & $\begin{array}{l}5 \\
1 \\
1\end{array}$ & $\begin{array}{l}1 \\
0 \\
1\end{array}$ \\
\hline Total & & 0 & 0 & 0 & 0 & 0 & 0 & 11 & 99 & 504 & 288 & 20 & 3 & 1 & 7 & 2 \\
\hline
\end{tabular}


Table 3. Cumulative Percentages of Matches of Each Week's Proofslips Received with Each Additional MARC II Tape Index

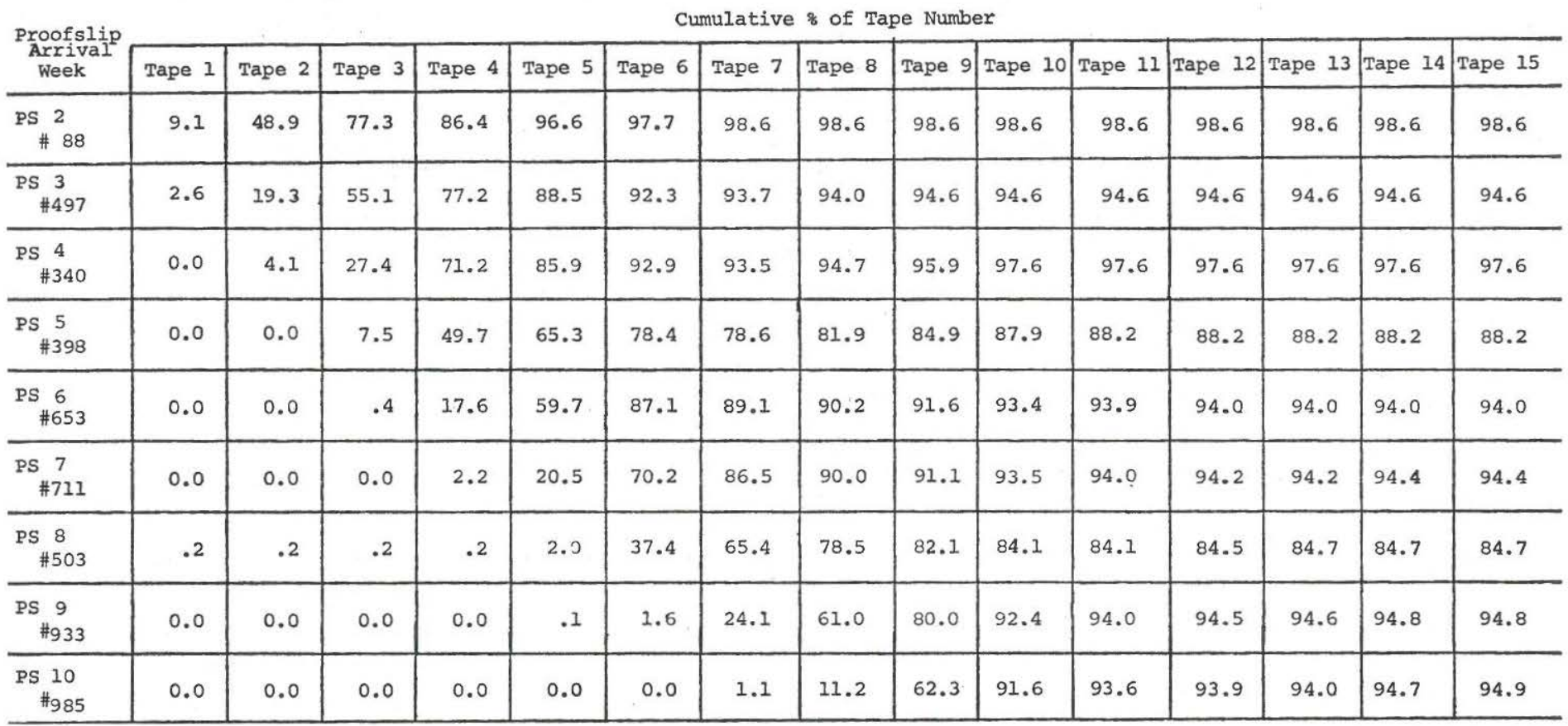


Table 4. Cumulative Percentages of Matches of Each MARC II Tape Index with Each Additional Week's Proofslips Received

\begin{tabular}{|c|c|c|c|c|c|c|c|c|c|c|c|c|c|c|}
\hline \multirow{2}{*}{$\begin{array}{c}\text { Tape } \\
\text { Number }\end{array}$} & \multicolumn{14}{|c|}{ Cumulative $s$ of Proofslip Week } \\
\hline & PS 2 & PS 3 & PS 4 & PS 5 & PS 6 & PS 7 & PS 8 & PS 9 & PS 10 & PS 11 & PS 12 & PS 13 & PS 14 & PS 15 \\
\hline $\begin{array}{l}\text { Tape } 1 \\
\# 648\end{array}$ & 1.2 & 3.2 & 3.2 & 3.2 & 3.2 & 3.2 & 3.4 & 3.4 & 3.4 & 3.4 & 3.4 & 3.4 & 3.4 & 3.4 \\
\hline $\begin{array}{l}\text { Tape } 2 \\
\# 495\end{array}$ & 7.1 & 23.8 & 26.6 & 26.6 & 26.6 & 26.6 & 26.6 & 26.6 & 26.6 & 26.6 & 26.6 & 26.6 & 26.6 & 26.6 \\
\hline $\begin{array}{l}\text { Tape } 3 \\
\# 494\end{array}$ & .5 .1 & 41.1 & 57.1 & 63.2 & 63.7 & 63.7 & 63.7 & 63.7 & 63.7 & 63.7 & 63.7 & 63.7 & 63.7 & 63.7 \\
\hline $\begin{array}{l}\text { Tape } 4 \\
\# 791\end{array}$ & 1.0 & 14.9 & 33.7 & 54.7 & 67.9 & 71.0 & 71.0 & 71.0 & 71.0 & 71.0 & 71.0 & 71.0 & 71.0 & 71.0 \\
\hline $\begin{array}{l}\text { Tape } 5 \\
\# 768\end{array}$ & 1.2 & 8.3. & 14.8 & 23.0 & 58.8 & 75.6 & 77.0 & 77.1 & 77.1 & 77.1 & 77.1 & 77.1 & 77.1 & 77.1 \\
\hline $\begin{array}{l}\text { Tape } 6 \\
\# 1136\end{array}$ & 0.1 & 1.9 & 4.0 & 8.5 & 24.2 & 55.4 & 71.0 & 72.3 & 72.3 & 72.4 & 72.4 & 72.4 & 72.4 & 72.4 \\
\hline $\begin{array}{l}\text { Tape } 7 \\
\# 694\end{array}$ & 0.1 & 1.2 & 1.4 & 1.6 & 3.5 & 20.2 & 40.5 & 70.7 & 72.3 & 72.6 & 72.6 & 72.6 & 72.6 & 72.6 \\
\hline $\begin{array}{l}\text { Tape } 8 \\
\# 658\end{array}$ & 0.0 & 0.2 & 0.7 & 2.7 & 3.8 & 7.6 & 17.6 & 69.9 & 84.9 & 86.2 & 86.5 & 86.5 & 86.5 & 86.5 \\
\hline $\begin{array}{l}\text { Tape } 9 \\
\# 892\end{array}$ & 0.0 & 0.3 & 0.8 & 2.1 & 3.1 & 4.0 & 6.1 & 26.0 & 82.5 & 86.0 & 86.3 & 86.3 & 86.3 & 86.3 \\
\hline $\begin{array}{l}\text { Tape } 10 \\
\# 922\end{array}$ & 0.0 & 0.0 & 0.7 & 2.0 & 3.3 & 5.0 & 6.2 & 18.5 & 49.8 & 79.5 & 89.9 & 89.9 & 90.5 & 90.5 \\
\hline
\end{tabular}




\section{CONCLUSION}

The data collected to date indicate that the arrivals of MARC records generally precede those of the corresponding proofslips. Thus, MARC records seem to be timely enough to be used in book selection and ordering processes, where proofslips are now used, as well as to supply bibliographic data for cataloging. 